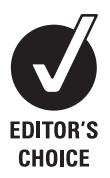

${ }^{1}$ University Department of Paediatrics, Addenbrooke's Hospital, Cambridge, UK;

${ }^{2}$ WellChild Laboratory, King's College London, Guy's Hospital, London, UK

Correspondence to: Professor David B Dunger, University Department of Paediatrics, Box 116, Level 8, Addenbrooke's Hospital, Hills Road, Cambridge CB2 200, UK: dbd25@cam.ac.uk

Accepted 16 December 2008 Published Online First 15 January 2009

\title{
Unchanged incidence of microalbuminuria in children with type 1 diabetes since 1986: a UK based inception cohort
}

\author{
R Amin, ${ }^{1}$ B Widmer, ${ }^{1}$ R N Dalton, ${ }^{2}$ D B Dunger ${ }^{1}$
}

\begin{abstract}
Aims: To prospectively determine the change in prevalence of microalbuminuria in relation to changes in glycaemic control in children diagnosed with type 1 diabetes between 1986 and 1996.

Design: Prospective observational study of an inception cohort.

Setting: The geographically defined region of Oxfordshire, UK.

Patients: 527 children diagnosed with type 1 diabetes under 16 years of age, were divided into three groups based on year of diagnosis of diabetes: group A (19861989, $n=165)$, group $B(1990-1993, n=179)$ and group C (1994-1996, $n=183)$. Each group was followed prospectively for 10 years.
\end{abstract}

Main outcome measures: Cumulative prevalence of microalbuminuria.

Results: After 4052 patient years of follow-up, in groups $C$ versus $B$ versus $A$, the cumulative prevalence of microalbuminuria was $31.7 \%(95 \% \mathrm{Cl} 20.9$ to 42.5$)$, $24.8 \%$ (17.8 to 31.8) and 23.2\% (15.4 to 30.0) (log rank $\mathrm{p}=0.22)$, and risk for development of microalbuminuria was not associated with year of onset of diabetes (hazard ratio 1.05 (0.99 to 1.12), $p=0.11$ ). In groups $C$ versus $B$ versus $A$, glycaemic control improved after 10 years of diabetes (mean HbA1c $8.9 \%$ (1.5\%) vs $9.4 \%$ (1.5\%) vs $10.1 \%(1.7 \%)$, p value for ANOVA $<0.001$ ) and more children achieved an $\mathrm{HbA} 1 \mathrm{c}$ level $<7.5 \%$ (15.6\% vs $5.9 \%$ vs $6.1 \%$, p value for ANOVA $=0.032$ )

Conclusion: In this UK based inception cohort of children diagnosed with type 1 diabetes, the adjusted prevalence of microalbuminuria was unchanged since 1986, despite some improvements in glycaemic control. This observation highlights the need for more proactive intervention with drugs such as angiotensin converting enzyme (ACE) inhibitors.

In adults with type 1 diabetes, the cumulative prevalence of microalbuminuria, an early marker for the development of diabetic nephropathy, ${ }^{1}$ is around $30 \%{ }^{2}$ Interventions to improve glycaemic control and lower blood pressure, and in particular the use of angiotensin converting enzyme (ACE) inhibitors reduce the risk for microalbuminuria ${ }^{3}$; consequently, observational studies have shown recent trends toward a reduced incidence of microalbuminuria. ${ }^{5}$

In those with childhood onset type 1 diabetes, the cumulative prevalence of microalbuminuria is greater than that observed in comparable adults cohorts after a similar duration of diabetes $\left(51 \%{ }^{6}\right.$ vs $30 \%^{2}$ after approximately 20 years), whereas the prevalence of clinical proteinuria is similar

\section{What is already known on this topic}

- Microalbuminuria is predictive of diabetic nephropathy and prevalence is greater in subjects with childhood onset compared with adult onset type 1 diabetes.

- Reduced prevalence of microalbuminuria has been reported in non-UK based cohorts.

\section{What this study adds}

- In the UK, the prevalence of microalbuminuria in childhood onset type 1 diabetes has been unchanged since 1986.

- There are trends towards improvements in $\mathrm{HbA1c}$ levels, although overall glycaemic control still remains poor and national targets are not being achieved.

(approximately 14\%) but occurs at a much younger age. ${ }^{6}$ Data from childhood onset cohorts from Europe and Australia show trends toward a reduced incidence of microalbuminuria. ${ }^{7-9}$ However, one study was cross-sectional ${ }^{8}$ and all were clinic rather than population based studies. It is important to relate these observations to glycaemic control, the main modifiable risk factor for microalbuminuria. One study showed no improvement in $\mathrm{HbA} 1 \mathrm{c}$ levels during follow-up, another showed improvement, ${ }^{9}$ while in the third study HbA1c levels were not reported. ${ }^{7}$

Reports of differences in HbA1c levels in different populations of children with type 1 diabetes reveal that achieving good control may involve as yet undetermined differences between centres. The Hvidore Study Group on Childhood Diabetes reported substantial differences in HbA1c levels between 22 European centres: some centres achieved excellent control, while others, particularly centres in the UK, had poor control with mean HbA1c levels above $9.2 \%{ }^{10}$ The reasons for these differences were unclear and are not completely explained by frequency of intensive insulin therapy use, diet and social differences. ${ }^{11}$ Consistent with these observations, data from the UK National Diabetes Audit 2004/5 showed that only $16 \%$ of children were achieving the national target of an $\mathrm{HbA1c}$ level of less than $7.5 \%{ }^{12}$ 
The aim of our study was to evaluate changes in microalbuminuria prevalence in relation to glycaemic control, in an inception cohort of UK based subjects with childhood onset type 1 diabetes recruited between 1986 and 1996 and followed for 10 years.

\section{METHODS \\ The Oxford Regional Prospective Study (ORPS) cohort}

The ORPS cohort was established in 1986. ${ }^{13}$ Children aged less than 16 years in the Oxford Health Authority area in the UK were identified over a 10-year period from the St Bartholomew's-Oxford diabetes register and were recruited within 3 months of diagnosis of diabetes. Case ascertainment for the register has been verified to be over $95 \% .{ }^{14}$ In total, $91 \%(\mathrm{n}=527)$ of eligible subjects were recruited between 1986 and 1997 at a mean age of 8.8 (SD 4.0) years. The dropout rate to date is $9.6 \%$. Ethics approval was obtained from district ethics committees, written consent from parents and verbal assent from children. Only $4 \%$ of the cohort has had less than 3 years of follow-up, while $9 \%$ have greater than 15 years of follow-up. In agreement with individual physicians, microalbuminuria was treated with ACE inhibitors or $\beta$-blockers if the subject was more than 18 years old and microalbuminuria was persistent or accompanied by hypertension.

For the purpose of this investigation, the cohort was divided into three groups based on the year of onset of type 1 diabetes: group $A$, 1986-1989 ( $\mathrm{n}=165)$; group $\mathrm{B}, 1990-1993(\mathrm{n}=179)$ and group $C$, 1994-1996 ( $n=183$ ). Each group was followed prospectively for a mean of 10 (SD 3.6) years. We grouped the participants into three cohorts (rather than analyse date of diagnosis as a continuous variable) in order to allow comparison with previously published data, ${ }^{8}$ where similar analyses were used.

\section{Annual assessments}

Subjects were assessed annually from the first year of diagnosis for height, weight and blood pressure and three consecutive early morning (first void) urine specimens were collected for the measurement of albumin:creatinine ratio (ACR). Blood samples were collected for centralised measurement of HbA1c.

\section{Definition of microalbuminuria}

Microalbuminuria was defined as an ACR of $3.5-35 \mathrm{mg} / \mathrm{mmol}$ in males and $4.0-47 \mathrm{mg} / \mathrm{mmol}$ in females in two out of three consecutive early morning urine collections during the annual assessment. This corresponded to an albumin excretion rate of $20-200 \mu \mathrm{g} / \mathrm{min}$, based on linear regression equations giving the relationship between ACR and albumin excretion rate measure in 304 timed overnight urine collections in a subgroup of patients. ${ }^{13}$ Three urine samples were collected in $84 \%$ of all annual assessments and two samples were collected in $16 \%$. Where only two urine samples were obtained, both had to be above the threshold to define microalbuminuria.

\section{Albumin assay}

Until 1994 urine samples were stored at $-20^{\circ} \mathrm{C}$ and after this at $-70^{\circ} \mathrm{C}$. Albumin was measured centrally by a double antibody ELISA method. The within and in-between assay coefficients of variation $(\mathrm{CV})$ were $6 \%$ and $12 \%$, respectively.

\section{Creatinine}

Creatinine was measured using a modified Jaffe method (Unimate 7, Roche Diagnostic Systems, Basel, Switzerland) on a Cobas Mira (Roche Diagnostic Systems) automated spectrophotometer. The CV was $2 \%$ at $2.2 \mathrm{mmol} / 1$.

\section{HbA1c}

Glycated haemoglobin was centrally measured initially by an electrophoretic method (Ciba Corning Diagnostics, Halstead, UK) and then by high performance liquid chromatography (DIAMAT; Bio-Rad, Hemel Hempstead, UK). The relationship between the two methods was carefully evaluated and has been described previously. ${ }^{13}$ The within-batch CV for the HPLC method was $2.2 \%$ and $1.3 \%$ at a level of $9.8 \%$ and $10.1 \%$, respectively. The between-batch CV was $3.5 \%$ and $2.2 \%$ at $5.6 \%$ and $10.1 \%$, respectively. The normal range was $4.3 \%$ to $6.0 \%$.

\section{Statistical methods}

As excretion of urinary albumin varies greatly within individuals and three consecutive measurements tend not to be normally distributed, we averaged three results from each subject with the geometric mean. The distributions of these yearly geometric means for the whole cohort were not normally distributed and were log transformed and analysed on this scale. This geometric mean from three samples was used for the annual urine result irrespective of the pre-defined microalbuminuria status. Other data were normally distributed. For each subject, data were summarised as means and data from the three groups (according to date of diagnosis of diabetes) were compared using an ANOVA model and $\chi^{2}$ analyses. In addition, the independent $t$ test and $\chi^{2}$ analyses were used to compare data from those with and without microalbuminuria. A life table method was used to calculate the cumulative prevalence of microalbuminuria after 10 years of diabetes. An ANOVA model was also used to compare adjusted measures of association with log urine ACR.

To display longitudinal changes in urine $\mathrm{HbA1c}$ levels, we used multilevel modelling software (MLwiN v 1.0 beta, Institute of Education, London, UK). This is a hierarchical regression model, allowing consideration of an individual's longitudinal data and summation by predefined groups. Variance in $\mathrm{HbA1c}$ was modelled against duration of diabetes. To recognise longitudinal HbA1c levels for each child, two levels of hierarchy in the data were used: level 1 consisted of all measurement points, and level 2 consisted of unique codes for each child. Models with the categorical explanatory variable (date of diagnosis of diabetes three groups) were built separately, and from these models graphs of the predicted values were drawn. A model was estimated separately for each of the three groups, and from these models we obtained a graph of the curves of mean predicted values over time in each group and standard errors of the mean. Each model was a hierarchical random-effects model, with level 1 of the data hierarchy being the measurement points nested within child as level 2, thereby allowing for the repeated measurements per child. $\mathrm{HbA1c}$ was modelled against diabetes duration using linear, quadratic and cubic terms and from these parameters we drew graphs of the mean ( \pm standard error of the mean) predicted values over time in each group. SPSS v 11.5 was used for the remainder of the statistical analyses and a $p$ value $<0.05$ was considered significant. Data are shown as mean (SD) unless otherwise stated.

\section{RESULTS}

\section{Characteristics of subjects with microalbuminuria}

After 10 years of diabetes and 4052 patient years of follow-up of 527 subjects, 110 patients developed microalbuminuria (20.9\%). The mean age of onset of microalbuminuria was 15.2 (3.9) years after 5.8 (2.7) years of diabetes. In subjects with microalbuminuria compared to those with normoalbuminuria, there were no differences in age at diagnosis of diabetes $(9.4$ (95\% CI 1.1 to $15.9)$ vs 8.7 ( 0.4 to 15.7 ) years, $p=0.11)$, current duration of 


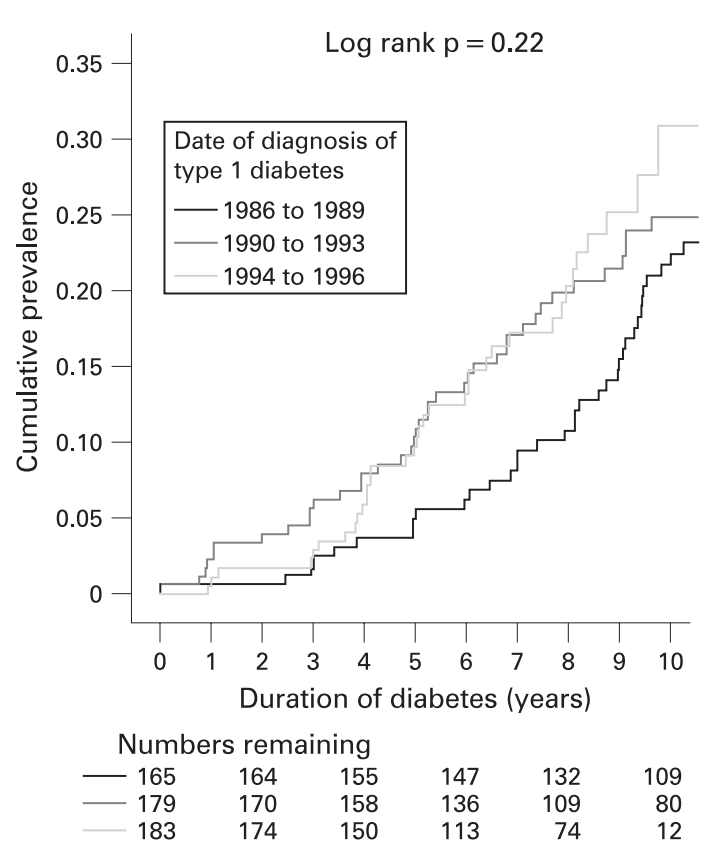

Figure 1 Kaplan-Meier survival curve showing the cumulative prevalence of developing microalbuminuria (110 events) in 527 patients with childhood onset type 1 diabetes after 10 years of diabetes, in relation to year of diabetes onset.

diabetes (9.8 (2.9 to 18.6) vs 9.8 (0.9 to 19.2$)$ years, $\mathrm{p}=0.99$ ) or current age (19.2 (8.4 to 28.6) vs 18.55 .7 to 31.8 ) years, $\mathrm{p}=0.14)$. Frequency of smoking history was no different $\left(\mathrm{n}=32(33.3 \%)\right.$ vs $\left.\mathrm{n}=103(25.0 \%), \chi^{2}=2.8, \mathrm{p}=0.10\right)$.

\section{Development of microalbuminuria in relation to date of diagnosis of diabetes}

The cumulative prevalence of microalbuminuria was no different between groups A (date of diagnosis 1986-1989), B (1990-1993) and C (1994-1996) (table 1 and fig 1). Microalbuminuria was not associated with year of onset of type 1 diabetes (hazard ratio 1.05 (0.99 to 1.12), $p=0.11$, after adjusting for known risk factors). Potential problems may arise if participants with a urine ACR near the lower limit of definition for microalbuminuria cross this level because of random measurement error or regression to the mean. Therefore, to improve sensitivity, we used an alternative cutoff for the definition of microalbuminuria. By reducing the lower limit by $0.5 \mathrm{mg} / \mathrm{mmol}, 119$ subjects met the criteria for microalbuminuria, but there was no difference in prevalence between the groups: group $\mathrm{A}, \mathrm{n}=36$ (21.9\%); group $\mathrm{B}, \mathrm{n}=41$
$(22.9 \%) ;$ and group C, $\mathrm{n}=42(22.7 \%) ; \chi^{2}=0.4, \mathrm{p}=0.97$. Similarly by reducing the lower limit for the definition of microalbuminuria by $1.0 \mathrm{mg} / \mathrm{mmol}, 129$ subjects met the criteria for microalbuminuria but there remained no difference between the groups: group $\mathrm{A}, \mathrm{n}=36(21.9 \%)$; group $\mathrm{B}, \mathrm{n}=45$ $(25.1 \%)$; and group $C, n=48(25.9 \%) ; \chi^{2}=1.1, p=0.66$. For further confirmation of these findings, in an ANOVA model, log urine ACR was not associated with date of diagnosis of diabetes $(\mathrm{F}=1.92, \mathrm{p}=0.15)$ after adjusting for known risk factors.

\section{Effects of glycaemic control}

Although overall glycaemic control was poor, for all three groups there was a trend towards lower HbA1c levels over time, particularly in group $\mathrm{C}$ (table 1 and fig 2). After 10 years of diabetes, mean $\mathrm{HbA} 1 \mathrm{c}$ levels were highest in group $\mathrm{A}$ and lowest in group $\mathrm{C}$ (table 1). Similarly the percentage of subjects with yearly $\mathrm{HbA1c}$ levels less than $9 \%$ and less than $7.5 \%$ after 10 years of diabetes was greatest in group $C$ and lowest in group A (group C vs B vs A: HbA1c $<9 \%, 58.4 \%$ vs $45.5 \%$ vs $29.5 \%$, $\chi^{2}=7.9, \mathrm{p}<0.001$ and $\mathrm{HbA} 1 \mathrm{c}<7.5 \%, 15.6 \%$ vs $5.9 \%$ vs $6.1 \%$, $\chi^{2}=7.9, p=0.032$ ) (fig 3). Of note, the greater percentage of subjects achieving an $\mathrm{HbA} 1 \mathrm{c}$ of $<7.5 \%$ in group $\mathrm{C}$ was only apparent in the final year of follow-up (fig 3).

In subjects with microalbuminuria compared to those with normoalbuminuria, mean HbA1c levels were higher $(10.7 \%$ $(1.7 \%)$ vs $9.6 \%(1.5 \%), p<0.001)$ and the percentage of subjects with a yearly $\mathrm{HbA1c}$ level $<7.5 \%$ was lower after 10 years of diabetes $\left(6.4 \%\right.$ vs $\left.11.8 \%, \chi^{2}=3.9, p=0.047\right)$.

\section{Intervention with anti-hypertensive medication}

Only $20(15 \%)$ subjects with microalbuminuria were started on ACE inhibitors or $\beta$-blockers for either persistent microalbuminuria and/or hypertension. Age at start of medication was 20.8 (4.1) years and median duration of diabetes was 11.2 (3.5) years. There was no statistical difference in HbA1c levels between subjects with microalbuminuria on medication compared to subjects with microalbuminuria but not on medication.

\section{DISCUSSION}

We report changes in the prevalence of microalbuminuria in relation to trends in glycaemic control over the previous 20 years in a large UK based, inception cohort of patients with childhood onset type 1 diabetes. The overall cumulative prevalence of microalbuminuria in our cohort is greater than previously reported in longitudinal childhood studies, ${ }^{15-17}$ but these previous studies were clinic based with variable diabetes duration at inclusion when compared with the current cohort, where all subjects were recruited at diagnosis and case

Table 1 Characteristics of the inception cohort of 527 patients with childhood onset type 1 diabetes after 10 years of diabetes, divided into three groups according to year of diagnosis of diabetes

\begin{tabular}{|c|c|c|c|c|}
\hline & \multicolumn{4}{|c|}{ Year of diagnosis of diabetes } \\
\hline & $\begin{array}{l}\text { Group A, } \\
1986-1989\end{array}$ & $\begin{array}{l}\text { Group B, } \\
\text { 1990-1993 }\end{array}$ & $\begin{array}{l}\text { Group C, } \\
\text { 1994-1996 }\end{array}$ & p Value \\
\hline Sample size, $\%$ male & $165(54)$ & $179(54)$ & $183(56)$ & \\
\hline Age at diagnosis diabetes, years & $8.8(4.1)$ & $8.6(4.2)$ & $9.2(3.8)$ & 0.31 \\
\hline Subjects with microalbuminuria, $\mathrm{n}(\%)$ & $35(19.0)$ & $39(20.9)$ & $36(19.1)$ & 0.87 \\
\hline Cumulative prevalence of microalbuminuria, $\%(95 \% \mathrm{CI})$ & $23.2(15.4$ to 30.0$)$ & $24.8(17.8$ to 31.8$)$ & $31.7(20.9$ to 42.5$)$ & 0.22 \\
\hline Mean $\mathrm{HbA} 1 \mathrm{c}$ in year 1 of diabetes, $\%$ & $10.3(2.1)$ & $10.2(2.0)$ & $9.6(2.1)$ & 0.09 \\
\hline Mean $\mathrm{HbA} 1 \mathrm{c}$ in year 10 of diabetes, $\%$ & $10.1(1.7)$ & $9.4(1.5)$ & $8.9(1.5)$ & $<0.001$ \\
\hline Insulin dose, units $/ \mathrm{kg} / \mathrm{day}$ & $0.9(0.2)$ & $0.9(0.3)$ & $0.9(0.3)$ & 0.33 \\
\hline
\end{tabular}

Data shown are mean (SD) unless otherwise stated. 
Figure 2 Yearly mean $\mathrm{HbA} 1 \mathrm{c}$ levels in 527 subjects with childhood onset type 1 diabetes, in relation to date of diagnosis of diabetes. Figures were derived from multilevel modelling (MLwiN v 1.0 beta, Institute of Education, UK). Dotted lines represent \pm standard error of the mean.

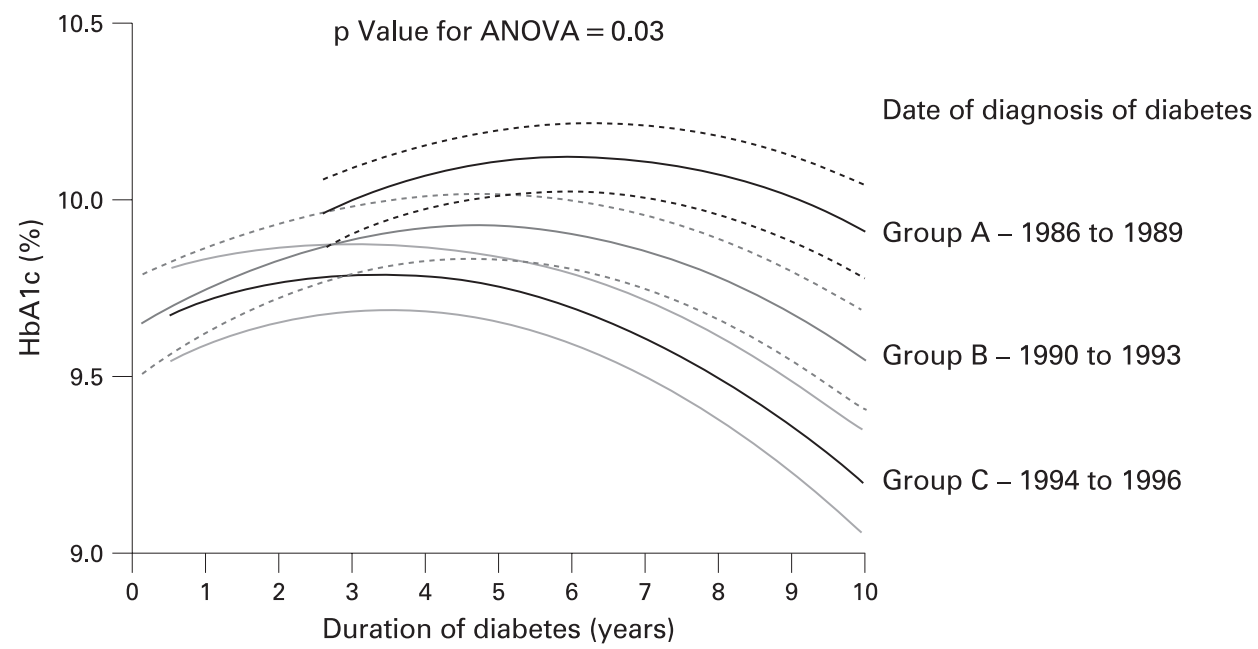

ascertainment was high with low dropout rates, indicating that observations from ORPS are an unbiased representation of care for childhood diabetes in the UK. ${ }^{6}$

The unchanged prevalence of microalbuminuria in our cohort over the last 20 years is in contrast with recent data from childhood onset cohorts from Sweden and Australia which showed a lower overall prevalence of microalbuminuria and significant trends toward reduced incidence in recent years. ${ }^{7-9}$ However, both these cohorts were clinic rather than population based, and case ascertainment was not reported. In addition, the Swedish definition of microalbuminuria was based initially on the urine dipstick test for proteinuria and subsequently on timed or spot collections of urine, with analysis of urine samples undertaken at numerous sites. ${ }^{79}$ In the Australian study, the definition of microalbuminuria was similar to that in our cohort, using three consecutive timed overnight collections of urine, but data were cross-sectional. ${ }^{8}$

If a true difference exists between these three international cohorts in changes in incidence of microalbuminuria, then this may relate to differences in glycaemic control, the most important modifiable risk factor for microalbuminuria. ${ }^{8}$ The overall mean HbA1c level for the Australian cohort was $8.6 \%$, for the Swedish cohort 7.1\%, and our cohort 9.7\%. All three studies used the HPLC method for measuring HbA1c for the

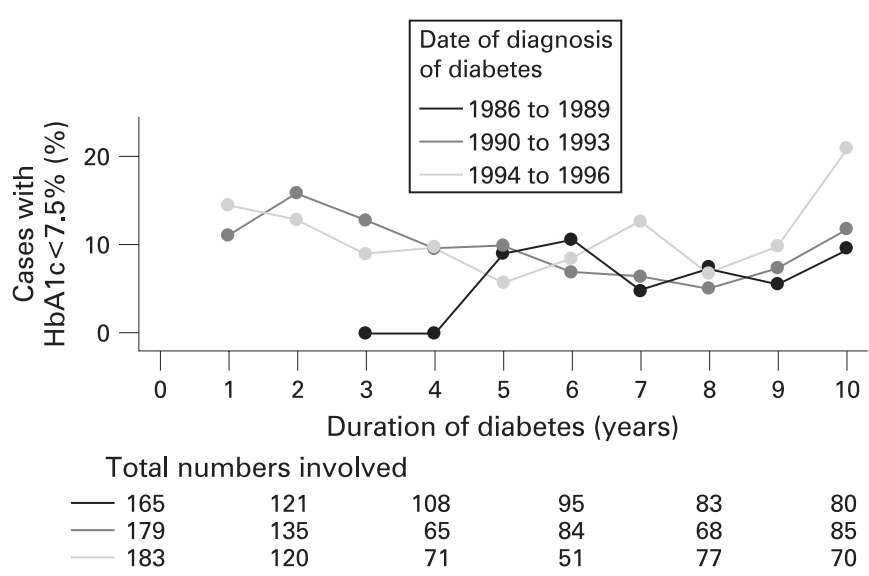

Figure 3 Percentage with yearly $\mathrm{HbA} 1 \mathrm{c}$ levels $<7.5 \%$ during the first 10 years of diabetes in 527 subjects with childhood onset type 1 diabetes, in relation to date of diagnosis of diabetes. majority of the study periods; however, laboratories in Sweden give, on average, readings $1 \%$ lower than in the Diabetic Control and Complications Trial. ${ }^{18}$ Even allowing for differences in assay, these variable $\mathrm{HbA1c}$ levels are in keeping with data from the Hvidore Study Group on Childhood Diabetes, which found substantial differences in central measurement of HbA1c levels in 22 European centres, with particularly poor control in the UK. ${ }^{10}$ These observations may relate to differences in factors such as communication, family structure and the medicalisation of adolescence within the medical culture. ${ }^{11}$

The observed differences in glycaemic control between European centres may also reflect differing medical practice, as for example, it is estimated that up to $20 \%$ of children with type 1 diabetes in Sweden are using insulin pump therapy. ${ }^{19}$ In the UK, estimates of pump use are much lower, although the use of basal bolus regimes and pump use in the ORPS cohort may have increased during the 10-year follow-up period of our study. However, importantly, the Hvidore Study Group showed no improvement in HbA1c levels with increased frequency of intensification therapy. ${ }^{20}$ Although there are other reports of improved glycaemic control in children using insulin pump therapy, ${ }^{21}{ }^{22}$ only a single randomised controlled trial exists, ${ }^{23}$ which was underpowered and found at the end of follow-up (only 12 months) that no difference existed in glycaemic control between those using insulin pump therapy and those on a basal bolus insulin regimen.

In our study, despite improvements in glycaemic control and numbers of subjects achieving HbA1c targets, the prevalence of microalbuminuria was unaffected, even when sensitivity analyses were evaluated using a lower cut-off level for the definition of microalbuminuria. Collectively these data add further weight to the growing body of evidence indicating that factors other than glycaemic control may be important in the pathogenesis of microalbuminuria during adolescence. ${ }^{24}{ }^{25}$ Nonmodifiable predictors of microalbuminuria in childhood include puberty and female sex,,$^{13}$ indicating that pubertal hormonal changes occurring in adolescents with type 1 diabetes may play a role. Consistent with this hypothesis, some experimental and clinical data have implicated changes in the growth hormone axis and also sex steroids in the development of pathogenic features characteristic of microalbuminuria, namely nephromegaly, glomerular hyperfiltration and basement membrane abnormalities. ${ }^{26}{ }^{27}$ A further complexity is that the development of diabetic nephropathy in type 1 diabetes maybe associated with familial risk for the development of type 2 diabetes, ${ }^{28}$ 
indicating the co-existence of an inherited insulin resistant component in the pathogenesis of microalbuminuria.

These data implicating other non-glycaemic genetic and environmental risk factors during adolescence suggest a more pragmatic approach to the prevention of microvascular complications should be considered. Intervention with ACE inhibitors in diabetic adults with microalbuminuria reduces the risk of progression to clinical proteinuria. ${ }^{4}$ For adolescents aged between 11 and 15 years of age, albumin excretion in the highest tertile of the normal range predicts $85 \%$ of subjects who go on to develop microalbuminuria and all of the subjects who develop clinical proteinuria. ${ }^{29}$ This together with the continued high incidence of microalbuminuria in the UK indicates that early renoprotection during puberty to prevent long term complications may be warranted. However, the decision to treat may be complicated as recent robust data from our group indicate that microalbuminuria is transient in $39 \%$ of cases, ${ }^{6}$ and only further follow-up will reveal whether there is later recurrence. In addition, factors such as compliance with long term therapy and unplanned pregnancies would need to be considered carefully. Although small reports show the likely efficacy of ACE inhibitors in adolescents, ${ }^{30-32}$ there is a need for a formal long term randomised controlled trial in this age group.

To conclude, the prognosis for childhood onset type 1 diabetes remains generally poor ${ }^{6}$ and the number of life years lost has remained unchanged over the last four decades. ${ }^{33}$ Consistent with these data, our UK based inception cohort of patients with childhood diabetes shows no change in the prevalence of microalbuminuria since 1986. This is despite a general improvement in glycaemic control, although only a minority of subjects are achieving national targets for $\mathrm{HbA1c}$ levels. A pragmatic rethink in strategies for earlier intervention to reduce urine albumin excretion is required in this age group.

Acknowledgements: We acknowledge The Juvenile Diabetes Research Foundation, the study field workers, the laboratory assistance of Angie Watts and Dot Harris, the Barts-0xford Study field workers, paediatricians, physicians and diabetes nurse specialists in the Oxford region. Members of the Oxford Regional Prospective Study Steering Committee are: DB Dunger, RN Dalton, J Fuller, EAM Gale, H Keen, M Murphy, HAW Niel, RJ Young and T Konopelska-Bahu. Members of the Oxford Regional Prospective Study are: J Edge, John Radcliffe Hospital, Oxford; HAW Neil and D Matthews, The Oxford Centre for Diabetes, Endocrinology and Metabolism, The Churchill Hospital, Oxford; RAF Bell and A Taylor, Horton General Hospital, Banbury; A Mukhtar, BP O'Malley, BR Silk and EH Smith, Kettering District Hospital, Kettering; RDM Scott, King Edward VII Hospital, Windsor; FM Ackland, CJ Fox and NK Griffin, Northampton General Hospital, Northampton; N Mann, H Simpson, P Cove Smith and M Pollitzer, Royal Berkshire Hospital, Reading; RS Brown and AH Knight, Stoke Mandeville Hospital, Aylesbury; JM Cowen and JC Pearce, Wexham Park Hospital, Slough.

Funding: The Oxford Regional Prospective Study is funded by Diabetes UK.

Competing interests: None.

Ethics approval: Ethics approval was obtained from district ethics committees.

\section{REFERENCES}

1. Viberti GC, Jarrett RJ, Keen H. Microalbuminuria as prediction of nephropathy in diabetics. Lancet 1982;2:611.

2. Hovind $\mathbf{P}$, Tarnow $L$, Rossing $P$, et al. Predictors for the development of microalbuminuria and macroalbuminuria in patients with type 1 diabetes: inception cohort study. BMJ 2004;328:1105.

3. Writing Team for the Diabetes Control and Complications Trial/Epidemiology of Diabetes Interventions and Complications Research Group. Sustained effect of intensive treatment of type 1 diabetes mellitus on development and progression of diabetic nephropathy: the Epidemiology of Diabetes Interventions and Complications (EDIC) study. JAMA 2003;290:2159-67.

4. ACE Inhibitors in Diabetic Nephropathy Trialist Group. Should all patients with type 1 diabetes mellitus and microalbuminuria receive angiotensin-converting enzyme inhibitors? A meta-analysis of individual patient data. Ann Intern Med 2001;134:370-9.

5. Bojestig M, Arnqvist HJ, Karlberg BE, et al. Unchanged incidence of severe retinopathy in a population of type 1 diabetic patients with marked reduction of nephropathy. Diabet Med 1998;15:863-9.
6. Amin R, Widmer B, Prevost AT, et al. Risk of microalbuminuria and progression to macroalbuminuria in a cohort with childhood onset type 1 diabetes: prospective observational study. BMJ 2008;336:697-701.

7. Nordwall M, Bojestig M, Arnqvist $\mathrm{HJ}$, et al. Declining incidence of severe retinopathy and persisting decrease of nephropathy in an unselected population of type 1 diabetes - the Linkoping Diabetes Complications Study. Diabetologia 2004:47:1266-72.

8. Mohsin F, Craig ME, Cusumano J, et al. Discordant trends in microvascular complications in adolescents with type 1 diabetes from 1990 to 2002. Diabetes Care 2005;28:1974-80.

9. Bojestig M, Arnqvist HJ, Hermansson G, et al. Declining incidence of nephropathy in insulin-dependent diabetes mellitus. N Engl J Med 1994;330:15-18.

10. Holl RW, Swift PG, Mortensen HB, et al. Insulin injection regimens and metabolic control in an international survey of adolescents with type 1 diabetes over 3 years: results from the Hvidore study group. Eur J Pediatr 2003;162:22-9.

11. Greene AC, Tripaldi M, Chiarelli F, et al. Cross-cultural differences in the management of children and adolescents with diabetes. Horm Res 2002;57/Suppl 1):75-7.

12. The Healthcare Commission. National Diabetes Audit. Report of the period 2004/5 London: The Healthcare Commission, 2006.

13. Schultz CJ, Konopelska-Bahu T, Dalton RN, et al. Microalbuminuria prevalence varies with age, sex, and puberty in children with type 1 diabetes followed from diagnosis in a longitudinal study. Oxford Regional Prospective Study Group. Diabetes Care 1999;22:495-502.

14. Gardner SG, Bingley PJ, Sawtell PA, et al. Rising incidence of insulin dependent diabetes in children aged under 5 years in the Oxford region: time trend analysis. The Bart's-Oxford Study Group. BMJ 1997:315:713-17.

15. Olsen BS, Johannesen J, Sjølie AK, et al. Metabolic control and prevalence of microvascular complications in young Danish patients with type 1 diabetes mellitus. Danish Study Group of Diabetes in Childhood. Diabet Med 1999:16:79-85.

16. Jones CA, Leese GP, Kerr S, et al. Development and progression of microalbuminuria in a clinic sample of patients with insulin dependent diabetes mellitus. Arch Dis Child 1998;78:518-23.

17. Rudberg S, Ullman E, Dahlquist G. Relationship between early metabolic control and the development of microalbuminuria - a longitudinal study in children with type 1 (insulin-dependent) diabetes mellitus. Diabetologia 1993;36:1309-14.

18. Kullberg CE, Bergstrom A, Dinesen B, et al. Comparisons of studies on diabetic complications hampered by differences in $\mathrm{GHb}$ measurements. Diabetes Care 1996;19:726-9.

19. Lindblad B. Data from the National Swedish Childhood Diabetes Registry. See http://www.ndr.nu (accessed 28 January 2009).

20. Danne T, Mortensen HB, Hougaard P, et al. Persistent differences among centers over 3 years in glycemic control and hypoglycemia in a study of 3,805 children and adolescents with type 1 diabetes from the Hvidore Study Group. Diabetes Care 2001;24:1342-7.

21. Litton J, Rice A, Friedman $\mathrm{N}$, et al. Insulin pump therapy in toddlers and preschool children with type 1 diabetes mellitus. J Pediatr 2002;141:490-5.

22. Weinzimer SA, Ahern JH, Doyle EA, et al. Persistence of benefits of continuous subcutaneous insulin infusion in very young children with type 1 diabetes: a follow-up report. Pediatrics 2004;114:1601-5

23. Doyle EA, Weinzimer SA, Steffen AT, et al. A randomized, prospective trial comparing the efficacy of continuous subcutaneous insulin infusion with multiple daily injections using insulin glargine. Diabetes Care 2004;27:1554-8.

24. Amiel SA, Sherwin RS, Simonson DC, et al. Impaired insulin action in puberty. A contributing factor to poor glycemic control in adolescents with diabetes. N Eng/ J Med 1986;315:215-19.

25. Amin R, Williams RM, Frystyk J, et al. Increasing urine albumin excretion is associated with growth hormone hypersecretion and reduced clearance of insulin in adolescents and young adults with type 1 diabetes: the Oxford Regional Prospective Study. Clin Endocrinol (Oxf) 2005;62:137-44

26. Amin R, Turner C, Van Aken S, et al. The relationship between microalbuminuria and glomerular filtration rate in young type 1 diabetic subjects: the Oxford Regional Prospective Study. Kidney Int 2005;68:1740-9.

27. Flyvbjerg A. Putative pathophysiological role of growth factors and cytokines in experimental diabetic kidney disease. Diabetologia 2000;43:1205-23.

28. Yip J, Mattock M, Sethi M, et al. Insulin resistance in family members of insulindependent diabetic patients with microalbuminuria. Lancet 1993;341:369-70.

29. Dunger DB, Schwarze CP, Cooper JD, et al. Can we identify adolescents at high risk for nephropathy before the development of microalbuminuria? Diabet Med 2007;24:131-6.

30. Drummond K, Levy-Marchal C, Laborde K, et al. Enalapril does not alter renal function in normotensive, normoalbuminuric, hyperfiltering type 1 (insulin-dependent) diabetic children. Diabetologia 1989;32:255-60.

31. Rudberg S, Østerby R, Bangstad HJ, et al. Effect of angiotensin converting enzyme inhibitor or beta blocker on glomerular structural changes in young microalbuminuric patients with type I (insulin-dependent) diabetes mellitus. Diabetologia 1999;42:589-95

32. Cook J, Daneman D, Spino M, et al. Angiotensin converting enzyme inhibitor therapy to decrease microalbuminuria in normotensive children with insulin-dependent diabetes mellitus. J Pediatr 1990;117(1 Pt 1):39-45.

33. Narayan KM, Boyle JP, Thompson TJ, et al. Lifetime risk for diabetes mellitus in the United States. JAMA 2003;290:1884-90. 